\title{
Pólipos vesiculares: benignos, malignos y cáncer de la vesícula biliar
}

\section{Gallbladder polyps: benign or malignant and gallbladder cancer}

Ricardo Oliveros-Wilches ${ }^{1 *}$ (1)

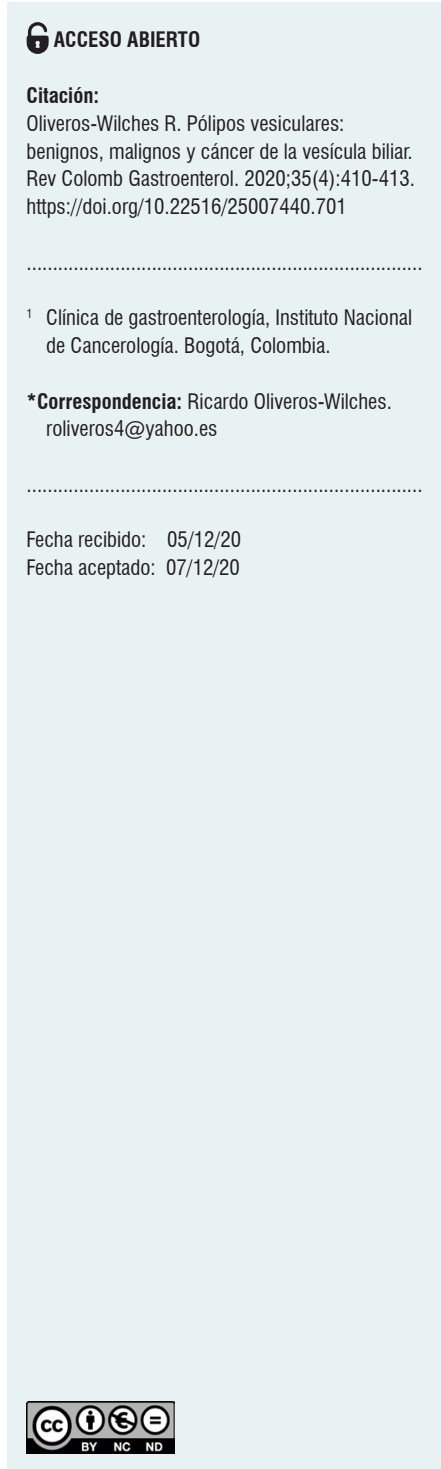

El carcinoma de la vesícula biliar (CVB) es el tumor maligno más común y agresivo de las vías biliares, con el período de sobrevida más corto; es altamente letal. En 2018, la Agencia Internacional para el Estudio del Cáncer (IARC) y Globocan estimaron que el CVB constituye el 1,7\% de todos los cánceres como causa de mortalidad, con 220000 casos nuevos diagnosticados anualmente (1).

El CVB es más frecuente en algunos sitios geográficos. La incidencia en el mundo varía substancialmente. Las mayores cifras se observan en Chile (27/100 000), seguidas por regiones del norte de India (21,5/100 000). En Chile (Suramérica), dentro de la comunidad de los indios mapuches de Valdivia, este tiene una incidencia en los hombres de 12,3 por cada 100000 habitantes y en las mujeres de 27,3 por cada 100000 habitantes $(2,3)$. Aunque es poco frecuente en algunas partes del mundo, es desproporcionadamente alta en algunos grupos étnicos $(1,3)$.

El CVB afecta más a las mujeres que a los hombres debido a que estas tienden a presentar más enfermedad vesicular por cálculos. Es uno de los pocos cánceres con una incidencia 3 a 6 veces mayor en las mujeres comparada con los hombres. Además, se incrementa con la edad, ya que se encontró que más de dos terceras partes de las personas diagnosticadas están por encima de los 65 años, con un promedio de 72 años (1).

Los factores asociados con mayores cifras de CVB son el sexo femenino, la localización geográfica, la etnicidad, la obesidad, una historia familiar o personal de cálculos vesiculares y la inflamación e infección crónica de la vesícula $(1,2,4)$.

El 70 \%-90 \% de los casos con CVB tienen historia de colelitiasis; sin embargo, es importante anotar que de la mayoría de las personas con colelitiasis solamente entre el 0,5\%-3 \% pueden desarrollar la enfermedad. El mecanismo exacto por el cual la colelitiasis desarrolla un carcinoma no es completamente entendido, y se piensa que la inflamación crónica y el daño irritativo del epitelio conlleva daño de la mucosa (1).

El tamaño de los cálculos contribuye al riesgo del CVB (4). Específicamente, los cálculos $>3 \mathrm{~cm}$ están asociados con un riesgo mayor ( 9,2 a 10,1 veces) en comparación con los cálculos $<1 \mathrm{~cm}(1)$. La inflamación persistente dentro de la vesícula parece contribuir a la calcificación de la pared, lo que da como resultado una vesícula en porcelana, que está asociada con un alto riesgo de desarrollo de cáncer (1).

La colangitis esclerosante (PSC) está también asociada con un incremento en el riesgo de CVB $(4,5)$. Anormalidades en la estructura del árbol biliar, específicamente de la unión del conducto pancreático con la vía biliar, hallazgos más prevalentes en la 
población asiática también se han asociado con un alto riesgo para el desarrollo de este carcinoma (1).

La inflamación y la irritación de la vesícula pueden originarse por infecciones bacterianas crónicas causadas comúnmente por la Salmonella typhi o el Helicobacter bilis. Los portadores de Salmonella tienen 12 veces más riesgo de desarrollar CVB; la infección crónica por la Salmonella es dos veces más común en las mujeres que en los hombres, mientras que el Helicobacter ha sido implicado en la formación de cálculos de colesterol (1).

Uno de los hallazgos más comunes en las imágenes de ultrasonido de la vesícula biliar son los pólipos y los cálculos. La presencia de pólipos en estos estudios puede resultar discordante con el resultado final de patología; la mayoría de los pólipos son benignos. Una de las metas de los trabajos es comparar las lesiones polipoides de la vesícula encontradas en las imágenes con el diagnóstico final de patología después de una colecistectomía $(4,5)$.

En este número se presenta un artículo sobre los factores de riesgo para malignidad de los pólipos vesiculares en dos hospitales públicos de Perú (6). Para ello, los autores recurren a los reportes de patología de las colecistectomías con un hallazgo y un diagnóstico confirmado de pólipos. Este es un trabajo retrospectivo que incluye 9 años de recolección de datos (2004-2012), en el que se encontraron 368 casos con un hallazgo en 26 (7\%) de pólipos con diagnóstico de adenocarcinoma y 341 (92,9\%) de pólipos benignos. Se incluyeron variables como la malignidad, la edad, el sexo, el tamaño del pólipo, el número de pólipos, la presencia de cálculos, el tamaño de la vesícula biliar y grosor de la pared vesicular; todos ellos factores que, en la práctica diaria, además del tamaño de los pólipos, ayudan a tomar decisiones clínicas, como definir la conveniencia de una colecistectomía $(4,5)$.

En el análisis bivariado se encontró que el tamaño del pólipo, el grosor de la pared vesicular, la litiasis vesicular, la edad del paciente y el tamaño de la vesícula estuvieron asociados con un mayor riesgo de malignidad del pólipo vesicular. El riesgo de malignidad se incrementó con el tamaño del pólipo y el grosor de la pared vesicular. Con relación al tamaño de los pólipos, los autores encontraron que los $>10 \mathrm{~mm}$ tienen un mayor riesgo de malignidad, pero también encontraron reportes de malignidad en pólipos $<10$ $\mathrm{mm}$, estableciendo el corte con una adecuada sensibilidad y especificidad para los $>6 \mathrm{~mm}$ de diámetro. La diferencia en el sexo y la presencia de cálculos no tuvo significancia como factor de riesgo.

El trabajo es muy relevante y se desarrolló muy bien debido a sus resultados estadísticos. Por supuesto, el artículo se relaciona con el diagnóstico de carcinoma incidental (estudio de patología), donde la literatura menciona que el carcinoma se encuentra entre el 0,2\%-1,1\% de todas las colecistectomías laparoscópicas (7).
El CVB se origina de la mucosa de la vesícula. El desarrollo temprano de este carcinoma es usualmente asintomático y tiene una alta propensión a la diseminación metastásica. Por esto, la mayoría de los pacientes son diagnosticados en estadios intermedios y avanzados, cuando ya no existe la posibilidad de un tratamiento curativo (1).

Mientras existe un consenso sobre la inflamación crónica como el evento patogénico inicial, el mecanismo de la progresión tumoral no está claramente establecido. Dos modelos han sido propuestos: metaplasia a displasia y adenoma a displasia (3). El modelo de metaplasia-carcinoma tiene una mayor aceptación, porque estas alteraciones histológicas son más frecuentes en comparación con los pólipos adenomatosos encontrados en menos del $3 \%$ de los especímenes obtenidos de los carcinomas tempranos $(3,4)$. Esta secuencia de metaplasia a displasia, a cáncer in situ y, finalmente, a cáncer invasivo puede durar cerca de 15 años (2).

Existe también un engrosamiento asimétrico inusual de la pared de la vesícula, con una infiltración de las estructuras vecinas. La mayoría de los carcinomas son adenocarcinomas ( $80 \%-95 \%)$, pudiendo ser papilares, tubulares mucinosos o con células en anillo de sello. Otros tipos de tumores con baja frecuencia son los carcinomas escamocelulares (16\%), carcinomas anaplásicos o indiferenciados (2\%$7 \%)$ y los carcinomas adenoescamosos (1\%-4\%). La mayoría de los tumores se encuentran en el fondo, cerca del $30 \%$ en el cuerpo y $10 \%$ en el cuello (2).

El CVB tiene una de las sobrevidas más cortas desde el momento del diagnóstico, lo que refleja su comportamiento agresivo y su diagnóstico tardío (7). Cabe destacar que solo 1 de 5 pacientes puede ser diagnosticado cuando la enfermedad está todavía localizada en la vesícula (1).

El CVB tiene una de las tres siguientes presentaciones:

1. En el diagnóstico de una enfermedad maligna (enfermedad avanzada).

2. En una enfermedad maligna detectada intraoperatoriamente por una colecistectomía aparentemente por enfermedad benigna.

3. En una enfermedad maligna diagnosticada de forma incidental en el examen patológico de una colecistectomía de rutina (4).

Se sospecha preoperatoriamente del carcinoma temprano de la vesícula biliar por imágenes en el 30 \%-40 \% de los casos y la mayoría son detectados de forma incidental en los exámenes histopatológicos de la vesícula (7).

Los pacientes (CVB) con carcinoma in situ tienen una sobrevida por encima del $80 \%$ a los 5 años, la cual declina a un $8 \%$ en los pacientes con estadios avanzados. Estas cifras demuestran la importancia de identificar los pólipos malignos y premalignos para permitir un tratamiento temprano y prevenir la diseminación del cáncer o el desarrollo de la 
malignidad. En pacientes con pólipos vesiculares verdaderos, la colecistectomía laparoscópica es la opción de tratamiento (4).

Los pólipos vesiculares (PV) tienen una prevalencia estimada del $5 \%$ en la población general, pero solamente el $5 \%$ de estos son considerados verdaderos pólipos vesiculares. La mayoría de los PV son detectados de forma incidental en las imágenes radiológicas o en el examen histológico después de una colecistectomía $(4,5)$.

Un número pequeño de pacientes con PV pueden ser sintomáticos y presentar cuadros de colecistitis aguda debido a la obstrucción del cístico por el pólipo, o por una colangitis debido a fragmentos del pólipo que pueden pasar al conducto biliar (5).

La mayoría de los pólipos vesiculares se clasifican como pseudopólipos (benignos), y corresponden a pólipos de colesterol, adenomiosis focal, pólipos hiperplásicos y pólipos inflamatorios; no tienen potencial maligno y no requieren seguimiento o intervención, mientras que los verdaderos pólipos, lo cuales incluyen los adenomas y adenocarcinomas, requieren un manejo quirúrgico. Los adenomas son benignos con potencial maligno y existe evidencia de la secuencia de adenoma-carcinoma como es vista en el cáncer colorrectal (CCR) (4). En pacientes con pólipos vesiculares verdaderos la colecistectomía laparoscópica es el mejor tratamiento (5).

Las imágenes radiológicas de la vesícula deben tener tres propósitos: ser capaces de diferenciar pólipos de cálculos o pliegues de la mucosa vesicular; diferenciar los verdaderos pólipos de los seudopólipos, y medir el tamaño de los pólipos de manera exacta, ya que actualmente es el factor más importante para definir si un paciente debe ir a una colecistectomía (4).

Existen factores de riesgo que pueden ayudar a definir la decisión clínica de una colecistectomía como el tamaño del pólipo, el número de pólipos, la morfología del pólipo, la presencia de cálculos, la edad, la procedencia del paciente (etnia), los antecedentes médicos (colangitis esclerosante primaria) y el grosor de la pared vesicular. (4).

Los estudios muestran que los pólipos malignos tienden a ser más grandes que los pólipos benignos $(5,8)$. Actualmente, el tamaño del pólipo en la imagen radiológica es el factor más determinante en el manejo de los pólipos vesiculares. Los estudios retrospectivos han encontrado que el riesgo de malignidad aumenta de forma importante de $10 \mathrm{~mm}$ hacia arriba, y el consenso general es que los pacientes con pólipos de $10 \mathrm{~mm}$ o de mayor tamaño deban ser tratados con una colecistectomía $(3,4)$.

Las guías publicadas por la Sociedad Europea de Radiología Gastrointestinal y Abdominal (ESGAR) respaldan esta conducta, pero dos revisiones sistemáticas $(4,9)$ muestran que mientras la mayoría de los pólipos malignos son de $10 \mathrm{~mm}$, existe un número significativo tanto de pólipos malignos o pólipos con potencial maligno por debajo de este umbral de tamaño. Hallazgos similares se encontraron en el artículo publicado en este número (6).

El principal determinante del riesgo de malignidad para la vesícula es el tamaño del pólipo (8); sin embargo, no todos los pólipos $<10 \mathrm{~mm}$ son benignos, razón por la cual es importante identificar factores de riesgo que permitan tener una mayor sospecha de malignidad y realizar la colecistectomía en pacientes con pólipos por debajo del límite de $10 \mathrm{~mm}$ (4).

La evidencia que muestra una mayor probabilidad de malignidad en los pólipos solitarios cuando se compara con los pólipos múltiples es muy débil. Bahtt y colaboradores (9) encontraron que la probabilidad en un pólipo $<10 \mathrm{~mm}$ y solitario era del $4,3 \%$. Por este resultado, la probabilidad de malignidad no es lo suficientemente alta para recomendar la colecistectomía en todos los pólipos solitarios. La sola presencia de un pólipo solitario debe ser considerada en combinación con otros factores de riesgo para malignidad (4), para así tomar una mejor decisión clínica con respecto a una colecistectomía.

Kwon y colaboradores (10) han demostrado que los pacientes con pólipos vesiculares de morfología sésil tienen un mayor riesgo de malignidad en comparación con los pólipos pedunculados (Odds ratio [OR]: 7,70 intervalo de confianza [IC] del $95 \% 2,48-23,95$ ). En otro trabajo (9), la probabilidad de malignidad fue del $13,9 \%$ en pólipos $<10$ $\mathrm{mm}$ y sésiles. Además, si el pólipo fue solitario y sésil, la probabilidad de malignidad fue del $24,8 \%$ y se recomendó una colecistectomía. La guía europea ESGAR utiliza la fortaleza de esta evidencia para recomendar la colecistectomía en todos los pacientes con pólipos vesiculares sésiles entre 6 y $9 \mathrm{~mm}$ de diámetro (4).

El riesgo de la mayoría de los cánceres se incrementa con la edad y un modelo similar es visto para el CVB. Varios artículos soportan este factor de la edad, pero el riesgo de malignidad varía significativamente entre los 50 y los 65 años (4).

Bhatt y colaboradores (9) encontraron que en los pacientes mayores de 50 años y con pólipos $<10 \mathrm{~mm}$ la probabilidad de malignidad fue del $20,7 \% \mathrm{y}$, por esto, se recomendó la colecistectomía. La ESGE utiliza esta evidencia para concluir que, si los pacientes son mayores de 50 años y los pólipos están entre 6 y $9 \mathrm{~mm}$ de diámetro, ellos deben realizarse una colecistectomía (4).

La evidencia de considerar la presencia de cálculos y de pólipos con mayor riesgo de malignidad es baja. En los pacientes sintomáticos debido a los cálculos, la colecistectomía está recomendada, y por esto la decisión es simple. Sin embargo, la evidencia no es tan fuerte para sugerir la colecistectomía en los casos con patología dual (pólipos y cálculos) (4). 
Un estudio (11) reporta un mayor riesgo de malignidad (13 veces más) en la población india, en comparación con la población caucásica (Hazard ratio [HR] 12,92 IC del $95 \%$ $3,77-44,29$ ) en un estudio con 5391 pacientes llevados a una colecistectomía. Este es el único estudio que compara el riesgo entre grupos étnicos. La ESGAR cree que este artículo es una evidencia suficiente, $y$ por eso recomienda que los pacientes de grupo étnico de la India con pólipos entre 6 a $9 \mathrm{~mm}$ de diámetro deben ser llevados a una colecistectomía (4).

La presencia de todos estos factores de riesgo mencionados en el artículo (6) y en la literatura pueden ayudar a tomar una decisión clínica sobre la colecistectomía, y también ayudan a disminuir el número de pólipos verdaderos omitidos en la categoría de $<10 \mathrm{~mm}$.

\section{REFERENCIAS}

1. Schmidt MA, Marcano-Bonilla L, Roberts LR. Gallbladder cancer: epidemiology and genetic risk associations. Chin Clin oncol 2019;8(4):31. https://doi.org/10.21037/cco.2019.08.13

2. Sharma A, Sharma K L, Gupta A, et al. Gallbladder cancer epidemiology, pathogenesis and molecular genetics: Recent update. World J Gastroenterol. 2017;23(22):3978-98. https://doi.org/10.3748/wjg.v23.i22.3978

3. Salazar M, Ituarte C, Abriata MG, et al. Gallbladder cancer in South America: epidemiology and prevention. Chin Clin Oncol 2019;8(4):32.

https://doi.org/10.21037/cco.2019.07.12

4. Wiles R, Thoeni R, Barbu ST, et al. Management and follow up of gallbladder polyps. Joint guidelines between the European Society of Gastrointestinal and abdominal Radiology (ESGAR) European Association for endoscopic Surgery and other interventional techniques (EAES), International Society of Digestive Surgery European Federation (EFISDS) and European Society of Gastrointestinal Endoscopy (ESGE). Eur Radiol. 2017;27(9):3856-66. https://doi.org/10.1007/s00330-017-4742-y

5. Li Y, Tejirian T, Collins J C. Gallbladder polyps: real or imagined? Am Surg. 2018;84(10):1670-74. https://doi.org/10.1177/000313481808401027
6. Mejía CR, Mayta K, Cárdenas M, Verástegui-Díaz A, Quiñones-Laveriano DM, Maravi-Coronado J, Monge E, Vera CA. Factores de riesgo para la malignidad de los pólipos vesiculares en dos hospitales públicos de Perú. Rev Colomb Gastroenterol. 2020;35(4):414-420. https://doi.org/10.22516/25007440.478

7. Acharya MR, Patkar S, Parray A, et al. Management of gallbladder cancer in India. Chin Clin Oncol 2019;8(4):35. https://doi.org/10.21037/cco.2019.07.03

8. Krell RW, Wei AC. Gallbladder cancer: surgical management. Chin Clin Oncol. 2019;8(4):36. https://doi.org/10.21037/cco.2019.06.06

9. Bhatt NR, Gillis A, Smoothey CO, et al. Evidence based management of polyps of the gallbladder. A systematic review of the risk factors of malignancy. Surgeon. 2016;14(5):278-86. https://doi.org/10.1016/j.surge.2015.12.001

10. Kwon W, Jang JY, Lee SE, et al. Clinicopathologic features of polypoid lesions of the gallbladder and risk factors of gallbladder cancer. J Korean Med Sci. 2009;24(3):481-87. https://doi.org/10.3346/jkms.2009.24.3.481

11. Aldouri AQ, MaliK HZ, Waytt J, et al. The risk of gallbladder cancer from polyps in a large multiethnic series. Eur J Surg Oncol. 2009;35(1):48-51. https://doi.org/10.1016/j.ejso.2008.01.036 